THORACIC SURGERY

\title{
Effect of training on patient outcomes following lobectomy
}

\author{
N Chaudhuri, A D Grayson, R Grainger, N K Mediratta, M H Carr, A S Soorae, \\ R D Page
}

See end of article for authors' affiliations ....................

Correspondence to: Mr R D Page, The Cardiothoracic Centre, Liverpool L1 4 3PE, UK: richard.page@ctc.nhs.uk

Received 16 May 2005 Accepted 11 January 2006 Published Online First 31 January 2006
Background: Little is known about the effect of surgical training on outcomes in thoracic surgery. The impact of surgeon training on outcomes following lung resection was examined, focusing on lobectomy as a marker operation.

Methods: 328 consecutive patients who underwent lobectomy at our institution between 1 October 2001 and 30 June 2003 were studied. Data were collected prospectively during the patient's admission as part of routine clinical practice and validated by a designated audit officer. Patient characteristics and postoperative outcomes were compared between trainee led and consultant led operations.

Results: In 115 cases (35.1\%) the operation was performed by a trainee thoracic surgeon as the first operator. There were no significant differences in patient characteristics between the two groups. Inhospital mortality was similar for operations led by trainees and consultants $(3.5 \%$ and $2.8 \%$, respectively; $\mathrm{p}>0.99$ ). Outcomes in the two groups did not differ significantly with respect to respiratory, cardiovascular, renal, neurological, chest infection, bleeding, and gastrointestinal complications. Survival rates at 1 year were $82.6 \%$ for procedures led by trainees compared with $81.7 \%$ for procedures led by consultants $(p=0.83)$.

Conclusions: With appropriate supervision, trainee thoracic surgeons can perform lobectomies safely without compromising short or intermediate term patient outcome.
A Il healthcare organisations have to balance between the need to provide a quality service while at the same time allowing for sufficient time for surgical training. ${ }^{1}$ With surgical performance under constant scrutiny, some consultant surgeons may be inclined to reduce the number of operations they allow a trainee to lead, which is at odds with the training requirements of junior surgeons. In addition, the new European Working Times Directive is reducing junior doctors' hours, thus leading to even less exposure and training opportunities.

Several studies have been published examining the impact of surgeon training on outcomes in cardiac surgery. ${ }^{2-6}$ In 2001 Jenkins and colleagues, in a study involving 6037 cardiac surgery operations, published their experience of surgical training and concluded that, with appropriate case selection, training in cardiac surgery can achieve good results. ${ }^{2}$ Our own institution recently published a report with similar conclusions. ${ }^{6}$ However, there are few data comparing trainee surgeons with their more experienced colleagues in thoracic surgery and very little is known about the outcomes.

The Society of Cardiothoracic Surgeons of Great Britain and Ireland has used lobectomy as a marker operation to assess perioperative outcomes of individual consultant surgeons. As it is the most common major thoracic surgical procedure performed, it forms the basis for thoracic surgical training. We therefore examined the impact of training on outcomes following lung resection, focusing on lobectomy as a marker operation.

\section{METHODS}

\section{Patient population and data}

A retrospective study of 328 consecutive patients undergoing lobectomy between 1 October 2001 and 30 June 2003 at the Cardiothoracic Centre-Liverpool was undertaken. All data were collected prospectively by the surgical teams during the patient admission as part of routine clinical practice and validated by a designated audit officer. The validation consisted of examining all entries in the thoracic surgery registry (Veritas Thoracic Version 2.0.04, Aurora Computer Services, 1998) and checking for completeness and consistency against the patients' medical records. Any inconsistencies were flagged back to the appropriate surgical team for clarification and amendments were made where applicable. All entries were also cross referenced against other sources in the hospital to ensure all cases had been captured.

Data collected included patient preoperative characteristics, lobe removed, in-hospital outcomes, and postoperative staging for primary tumours. Whether the lobectomy was performed with the trainee as first operator was also documented.

Chronic obstructive pulmonary disease (COPD) was defined as patients with a diagnosed history of COPD requiring use of bronchodilators or steroids. Current smokers were defined as patients smoking during the 2 months before surgery. In-hospital mortality was defined as death within the same hospital admission regardless of cause. Re-exploration for bleeding included any patient who required surgical reoperation after initial departure from the operating theatre. Respiratory complications included postoperative mechanical ventilation $>24$ hours or reintubation for ventilatory support after the day of surgery; pneumonia (defined as fever, leucocytosis pulmonary infiltrate requiring antibiotic therapy); air leak from thoracostomy tubes for more than 6 days postoperatively; lobar collapse on postoperative chest radiograph; empyema; and bronchopleural fistula. Cardiovascular complications included new requirement for pharmacological or other management of cardiac arrhythmias; need for

Abbreviations: $C A B G$, coronary artery bypass grafting; $C O P D$, chronic obstructive pulmonary disease; $\mathrm{FEV}_{1}$, forced expiratory volume in 1 second; FVC, forced vital capacity 
Table 1 Patient characteristics

\begin{tabular}{llll}
\hline & $\begin{array}{l}\text { Trainee led } \\
(\mathbf{n = 1 1 5 )}\end{array}$ & $\begin{array}{l}\text { Consultant led } \\
(\mathbf{n = 2 1 3 )}\end{array}$ & p value \\
\hline Age (years) & $70.1(60.9-75.9)$ & $68.7(61.7-73.8)$ & 0.51 \\
Female (\%) & $57.4(66)$ & $51.6(110)$ & 0.32 \\
FEV $(\%)$ & $76.1(61.5-90.9)$ & $75.4(61.3-87.3)$ & 0.38 \\
FVC (\%) & $88.4(78.1-101.6)$ & $87.6(73.6-98.5)$ & 0.22 \\
Current smokers (\%) & $31.3(36)$ & $33.8(72)$ & 0.64 \\
COPD (\%) & $21.7(25)$ & $23.5(50)$ & 0.72 \\
Non-elective surgery (\%) & $3.5(4)$ & $3.8(8)$ & 0.89 \\
Primary tumour (\%) & $94.8(109)$ & $95.3(203)$ & 0.84 \\
\hline Numbers of patients are shown in parentheses. & & \\
FEV, forced expiratory volume in 1 second; FVC, forced vital capacity; COPD, chronic obstructive pulmonary \\
disease. \\
Continuous variables shown as median values with 25th and 75th percentiles. \\
Categorical variables shown as a percentage.
\end{tabular}

postoperative inotropic support; pulmonary embolism; and myocardial infarction. A postoperative rise in serum creatinine above $200 \mu \mathrm{mol} / \mathrm{l}$ or a need for postoperative dialysis support in a patient with normal preoperative renal function were considered to be renal complications. A postoperative new cerebrovascular accident or transient ischaemic attack was considered to be a neurological complication. Postoperative gastrointestinal complications were in line with the definition of The Society of Cardiothoracic Surgeons of Great Britain and Ireland and included gastrointestinal bleeding, pancreatitis, ischaemic bowel, and perforation. ${ }^{7}$

\section{Patient follow up}

Patient records were linked to the National Strategic Tracing Service (NSTS) which records all deaths in the UK. To establish current vital status at 1 year, patients were matched to the NSTS based on patient name, National Health Service number, date of birth, sex, and postcode.

Approval was obtained from the hospital's academic committee.

\section{Surgical technique}

The standard surgical approach was a posterolateral thoracotomy through the fourth or fifth intercostal space. The pulmonary vessels were doubly ligated and divided in between. The bronchus was stapled and divided distally. The individual suture material and mechanical stapling device differed between consultant surgeons, but the surgical technique and approach to the lobectomy were similar. Two intercostal drains were inserted in all cases at the end of the procedure. An epidural catheter was used to administer a mix of $0.1 \%$ bupivacaine and $5 \mu \mathrm{g} / \mathrm{ml}$ fentanyl for postoperative analgesia unless contraindicated.

During a junior surgeon's training at the centre they are expected to perform thoracotomies, wedge lung resections, and other minor thoracic surgical procedures at the commencement of training, while assisting in the performance of

Table 2 Lobe removed

\begin{tabular}{lll}
\hline & $\begin{array}{l}\text { Trainee led } \\
\text { (n= 115) }\end{array}$ & $\begin{array}{l}\text { Consultant led } \\
\text { (n=213) }\end{array}$ \\
\hline Left upper (\%) & $25.2(29)$ & $29.6(63)$ \\
Left lower (\%) & $13.0(15)$ & $12.7(27)$ \\
Right upper (\%) & $40.9(47)$ & $37.6(80)$ \\
Right middle (\%) & $4.3(5)$ & $7.0(15)$ \\
Right lower (\%) & $16.6(19)$ & $13.1(28)$ \\
\hline
\end{tabular}

Numbers of patients shown in parentheses.

$\mathrm{p}=0.69$ ( $\chi^{2}$ test).

Categorical variables shown as a percentage. major lung resections. They then proceed to closely supervised segmentectomies, lobectomies, and pneumonectomies. As the trainees progress to their final years of surgical training they become independent operators. The consultant surgeon is always immediately available for advice or help in case of difficulties.

\section{Statistical analysis}

Continuous variables are shown as median values with 25 th and 75 th percentiles because of non-normality of data. Categorical variables are shown as a percentage. Comparisons were made using Wilcoxon rank sum tests and $\chi^{2}$ tests as appropriate. When appropriate, the Yates continuity correction was used. Deaths occurring as a function of time were described using the product limit methodology of Kaplan and Meier. ${ }^{8}$ In all cases a p value of $<0.05$ was considered significant. All statistical analysis was performed with SAS for Windows Version 8.2.

\section{RESULTS}

Of the 328 lobectomy operations undertaken during the study period, $115(35.1 \%)$ were performed by a trainee thoracic surgeon as first operator. A trainee surgeon in years $1-3$ of their training performed 73 operations and trainees in years 4-6 performed 42 operations. The characteristics of the patients based on whether the lobectomy was led by a trainee or by a consultant are shown in table 1 . No significant differences were found with respect to age, sex, respiratory function, smoking status, COPD, priority, and primary tumours between the two groups.

The different lobes removed by either trainee or consultant thoracic surgeons are shown in table 2. No significant difference was observed.

Table 3 shows in-hospital outcomes for trainee and consultant led lobectomy operations. The in-hospital mortality was similar for lobectomies led by trainee and consultant thoracic surgeons $(3.5 \% \vee 2.8 \%$; p $>0.99)$. No differences existed with respect to respiratory, chest infection, cardiovascular, renal, neurological, bleeding and gastrointestinal complications. The mean postoperative length of stay was also similar for the two surgeon groups.

There was a significant difference between trainee and consultant surgeons in the postoperative staging of primary tumours, with consultant led lobectomies being performed on higher staged patients $(\mathrm{p}=0.032$; table 4$)$.

Fifty nine deaths (18\%) occurred during the 1 year follow up period. Follow up was complete for all patients. No significant difference existed between the groups $(p=0.83)$. Survival at 1 year for trainee led procedures was $82.6 \%$ compared with $81.7 \%$ for procedures led by consultants (fig 1). 
Table 3 Postoperative outcomes

\begin{tabular}{llll} 
& $\begin{array}{l}\text { Trainee led } \\
(\mathbf{n}=115)\end{array}$ & $\begin{array}{l}\text { Consultant led } \\
(\mathbf{n}=213)\end{array}$ & p value \\
\hline In-hospital mortality (\%) & $3.5(4)$ & $2.8(6)$ & 0.74 \\
Respiratory complication (\%) & $13.0(15)$ & $16.4(35)$ & 0.42 \\
Infection complication (\%) & $3.5(4)$ & $4.2(9)$ & 0.74 \\
Cardiovascular complication (\%) & $8.7(10)$ & $8.9(19)$ & 0.94 \\
Renal complication (\%) & $3.5(4)$ & $4.2(9)$ & 0.74 \\
Neurological complication (\%) & $1.7(2)$ & $1.9(4)$ & 0.93 \\
Re-exploration for bleeding (\%) & $0(0)$ & $2.4(5)$ & 0.10 \\
Gastrointestinal complication (\%) & $1.7(2)$ & $0.9(2)$ & 0.53 \\
Length of stay (days) & $8(7-11)$ & $8(7-11)$ & 0.58 \\
\hline Numbers of patients shown in parentheses. & & \\
Continuous variables shown as median value with 25th and 75th percentiles. \\
Categorical variables shown as a percentage.
\end{tabular}

\section{DISCUSSION}

Increasing public scrutiny of the results of individual surgeons has led to greater caution among senior surgeons to provide training because of the general belief among clinicians that trainee surgeons are associated with increased morbidity and a prolonged hospital stay, and therefore increased hospital costs. A reduction in junior doctors' hours has also affected the time a surgeon in training spends in an operating theatre.

Surgical training in cardiac surgery has increasingly been shown to be safe. ${ }^{2-6}$ Data from our own institution have shown that, after adjusting for case mix, with careful case selection, training does not adversely affect the early and mid term outcomes of coronary artery bypass grafting (CABG). In-hospital morbidity and mortality results were similar between consultant and trainee surgeons but, equally important, the adjusted survival at 4 years was also not significantly different. ${ }^{6}$ Training in off pump CABG has also been shown to be safe with acceptable results. ${ }^{9}{ }^{10}$ However, no data have been published comparing the results of thoracic surgery led by consultant and trainee surgeons.

In our study trainees performed a significant proportion of the case load of lobectomies (35.1\%). The preoperative patient characteristics did not show any significant difference between the trainee led and consultant led cases. However, as most of the cases were a result of primary lung tumours, an analysis of the tumour stages revealed a clear case selection for training. Using higher TNM stages as a surrogate marker for the technical difficulty of a particular case, it was found that trainee surgeons were more likely to operate on patients with UICC TNM stage I or II tumours than consultant surgeons.

It is also generally believed that a lower lobectomy is usually technically easier than an upper lobectomy. However, there was no significant difference between consultants and trainees with regard to the proportions of particular lobe removed. Despite this lack of objective data, individual patients may have been selected as unsuitable for trainee led surgery based on subtle clinical reasons. These reasons are

Table 4 Postoperative staging in primary tumour

\begin{tabular}{cll}
\hline Tumour stage & $\begin{array}{l}\text { Trainee led } \\
(\mathbf{n}=109)\end{array}$ & $\begin{array}{l}\text { Consultant led } \\
(\mathbf{n}=203)\end{array}$ \\
\hline la (\%) & $31.1(34)$ & $30.0(61)$ \\
Ib (\%) & $51.4(56)$ & $36.0(73)$ \\
Ila $(\%)$ & $3.7(4)$ & $8.4(17)$ \\
Ilb (\%) & $10.1(11)$ & $12.8(26)$ \\
Illa (\%) & $3.7(4)$ & $12.8(26)$ \\
\hline
\end{tabular}

Numbers of patients shown in parentheses.

$\mathrm{p}=0.032\left(\chi^{2}\right.$ test for trend).

Categorical variables shown as a percentage. difficult to define in prospective as well as retrospective studies. The competence level of individual trainees also impacts on the suitability of a particular case for training. What is more significant is that, even if in a particular case the trainee is not the first operator, parts of the proceduresuch as thoracotomy and closure of the thoracotomy wound-may be used for training purposes. Again retrospective evaluation limits our ability to obtain such data, although in our institution we record the trainee as the primary surgeon on an intention to treat basis. However, prospective collection of data in our database will ensure the actual level of trainee participation in each individual case.

In our study the postoperative mortality and morbidity was not significantly different between consultant and trainee led cases. The postoperative length of stay was also similar for the two groups. There was therefore no significant difference in early postoperative patient outcomes between trainees and consultants. Survival up to l year after surgery followed almost identical paths in both groups (fig 1). Early survival patterns were similar in the consultant and trainee led groups and correlate well with national standards.

Our study has several limitations. Firstly, it is an observational study and could be biased by factors not recorded in our thoracic surgery registry. Another limitation is the fact that the results of trainee led procedures will probably partly reflect the performance of the consultant who will have provided assistance to the junior surgeon. Unfortunately, we cannot quantify the full extent of involvement by the consultants in this retrospective study. Also, as a result of the fairly recent reference time frame, long term survival data are not available for our cohort of patients. It is possible that long term survival may differ between consultant and trainee led procedures as a result of case selection.

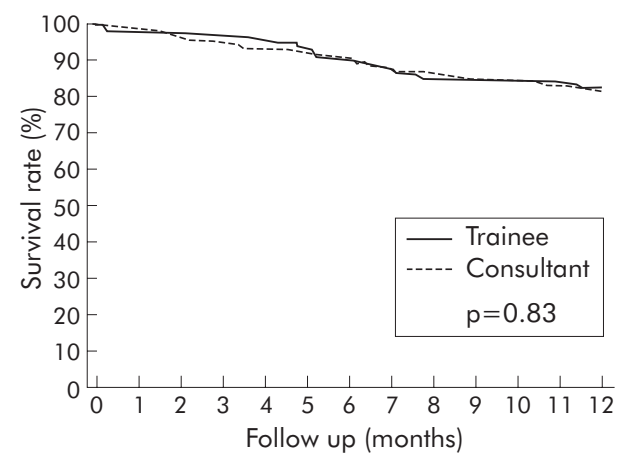

Figure 1 Survival rate following lobectomy operations led by trainee or consultant thoracic surgeons. 
With increasing scrutiny of surgical performance and the need for accountability not only in the UK but worldwide, it is important to audit surgical performance regularly and the same is true for trainee thoracic surgeons. However, adequate allowance must be made for differences in case mix, if they exist. There is a current trend to focus on the importance of wet-labs and simulation in surgical training. ${ }^{11}$ However, surgical skills require the integration of decision making and mechanical processes. ${ }^{12}$ Wet-labs are invaluable in imparting the basic surgical skills and maximising the trainee's time in acquiring and perfecting new techniques. Supervised training in the operating theatre, however, is exceedingly important to develop the decision making skills or strategy. Initially, the supervisor plays an active role in the operation and may occasionally have to take over from the trainee, especially if the patient's clinical condition or pathological anatomy deviates from the norm. As training progresses the trainee begins to anticipate such deviations and is able to develop his or her own strategy to manage such situations. The role of the trainer then becomes more passive. It is obviously of paramount importance that patients do not come to any harm by having a trainee operating on them while, at the same time, the trainee becomes better equipped to deal with real life situations for the benefit of his or her future surgical practice. Studies such as ours are important as they highlight this often ignored aspect of surgical procedures performed by trainees and should encourage supervised thoracic surgical training in the operating theatre.

In conclusion, with appropriate supervision, trainee thoracic surgeons can perform lobectomies safely and without compromising the results in the short or intermediate term. While a detailed prescription for training in thoracic surgery is beyond the scope of this article, the basic principles should include the acquisition and perfection of technical skills in a wet-lab or simulator with a simultaneous and gradual progression in supervised training within the operating theatre. There should be continuous feedback from the supervisor on the path to surgical competence.

Future prospective studies are essential to validate our conclusions and to collect objective data on the actual amount of supervision necessary in theatre for an individual trainee to attain technical and decision making competencies. Such studies should also attempt to delineate the exact role of wet-labs and simulators in parallel to training within the operating theatre.

\section{ACKNOWLEDGEMENTS}

The authors acknowledge the cooperation given by Mr M J Drakeley, consultant cardiothoracic surgeon.

\section{Authors' affiliations}

N Chaudhuri, N K Mediratta, M H Carr, A S Soorae, R D Page,

Department of Cardiothoracic Surgery, The Cardiothoracic Centre, Liverpool, UK

A D Grayson, R Grainger, Department of Clinical Governance, The Cardiothoracic Centre, Liverpool, UK

Funding: none.

Conflict of interest: none declared.

Contributorship: study concept and design (NC, ADG); study retrieval and data extraction (NC, ADG, RG); analysis and interpretation of data (NC, ADG, RG, ASS, RDP); drafting of manuscript (NC, ADG); critical revision of manuscript for important intellectual content (RG, NKM, MHC, ASS, RDP).

Presented at the 3rd EACTS-ESTS Joint Meeting, Leipzig, Germany, September 2004.

\section{REFERENCES}

1 Hargreaves DH. A training culture in surgery. BMJ 1996;313:1635-9.

2 Stoica SC, Sharples LD, Ahmed I, et al. Preoperative risk prediction and intraoperative events in cardiac surgery. Eur J Cardiothorac Surg 2002;21:41-6.

3 Goodwin AT, Birdi I, Ramesh TPJ, et al. Effect of surgical training on outcome and hospital costs in coronary surgery. Heart 2001;85:454-7.

4 Baskett RJF, Buth KJ, Legare JF, et al. Is it safe to train residents to perform cardiac surgery? Ann Thorac Surg 2002;74:1043-9.

5 Jenkins DP, Valencia O, Smith EE. Risk stratification for training in cardiac surgery. Thorac Cardiovasc Surg 2001;49:75-7.

6 Oo AY, Grayson AD, Rashid A. Effect of training on outcomes following coronary artery bypass graft surgery. Eur J Cardiothorac Surg 2004;25:591-6.

7 The Society of Cardiothoracic Surgeons of Great Britain and Ireland. National adult cardiac surgical database report 1999-2000, May, 2001.

8 Kaplan EL, Meier P. Nonparametric estimation from incomplete observations. J Am Stat Assoc 1958;53:547-81.

9 Caputo M, Bryan AJ, Capoun R, et al. The evolution of training in off-pump coronary surgery in a single institution. Ann Thorac Surg 2002;74:S1403-7.

10 Karamanoukian HL, Panos AL, Bergsland J, et al. Perspectives of a cardiac surgery resident in-training on off-pump coronary bypass operation. Ann Thorac Surg 2000;69:42-6.

11 Ramphal PS, Coore DN, Craven MP, et al. A high-fidelity tissue based cardiac surgical simulator. Eur J Cardiothorac Surg 2005;27:910-6.

12 Hamdorf JM, Hall JC. Acquiring surgical skills. Br J Surg 2000;87:28-37. 